\title{
Predicting Cognitive Decline and Dementia with the Newly Normed SKT Short Cognitive Performance Test
}

\author{
Mark Stemmler ${ }^{\mathrm{a}} \quad$ Johannes Baltasar Hessler $^{\mathrm{b}}$ Horst Bickel $^{\mathrm{b}}$ \\ a Department of Psychology, Friedrich-Alexander University of Erlangen-Nuremberg (FAU), \\ Erlangen, Germany; ${ }^{\mathrm{b}}$ Department of Psychiatry and Psychotherapy, Klinikum Rechts der Isar, \\ Technical University of Munich (TUM), Munich, Germany
}

\author{
Keywords \\ Cognitive tests - SKT (Syndrom Kurztest) - Predictive validity - Cox regression - Dementia · \\ Cognitive impairment $\cdot$ Mild cognitive impairment
}

\begin{abstract}
Objective: The aim of this article was to determine the criterion-related validity of the newly normed SKT (Syndrom-Kurztest) Short Cognitive Performance Test with the onset of dementia as the predicted criterion. Methods: The cognitive ability was tested with the SKT in a sample of 546 cognitively healthy adults aged $65-85$ years. New cases of mild cognitive impairment $(\mathrm{MCl})$ or dementia were determined in 3 follow-up investigations at 1-year intervals. Each participant's cognitive status was rated on the Clinical Dementia Rating Scale. The cognitive status according to the SKT is presented in terms of a traffic light system. Results: Based on Kaplan-Meier estimators, the trajectories of the different SKT traffic light labels were investigated over 3 years. The trajectories were significantly different, representing differential risks for dementia onset. In comparison to the green group, the hazard ratio (HR) for the development of dementia and $\mathrm{MCl}$ amounted to $\mathrm{HR} 6.63$ (95\% Cl 2.75-15.96) and HR 2.34 (95\% $\mathrm{Cl}$ 1.37-3.99), respectively, in the yellow group, and to $\mathrm{HR} 25.40$ (95\% Cl 10.73-60.14) and HR 3.83 (95\% Cl 1.86-7.86), respectively, in the red group. Conclusions: The newly normed SKT showed a high predictive validity for the onset of dementia.




\section{Introduction}

The assessment of cognitive decline is an important and necessary first step of the diagnostic procedure for the assessment of dementia due to Alzheimer's disease or any other dementia type $[1,2]$. Next to assessing the degree of cognitive impairment, it is desirable to have measurement tools that are able to predict the cognitive deterioration of an older adult in the future. A cognitive test with high predictive or criterion-related validity [3] may help to predict the onset of clinical dementia during preclinical and mild cognitive impairment $(\mathrm{MCI})$ stages [4,5], which often last several years [6]. Once recognized, these persons could be monitored more closely in the following time, allowing them to initiate personal and medical measures in time.

The present study investigated the predictive validity of the SKT Short Cognitive Performance Test (Syndrom-Kurztest [SKT]) [7], a short test for the detection of early cognitive decline in older persons. The SKT was newly normed in 2015 based on continuous regressions [8]; the testing material was not changed. The old norms of the SKT from 2001 had to be renewed because reports from clinicians suggested that the old norms were too lenient at the early stages of cognitive decline and lead to many false negatives [9]. In a crosssectional study, the new norms showed better sensitivity than the old norms [10]. For the comparison of healthy cognitive development versus MCI, the sensitivity was 0.89 (old norming: 0.65); for the comparison of no dementia versus dementia, it was 0.83 (old norming: 0.78) and for the comparison between MCI versus dementia it was 0.83 (old norming: 0.78). In comparison to other well established cognitive tests, the SKT has a number of advantages or unique characteristics: (1) there exist 5 parallel forms of the SKT (form A through E), in order to test the cognitive development of the same subjects over time; this is especially important if subjects with MCI subjects or older adults affected by mild dementia are repeatedly tested which avoids retest and memory effects. (2) The testing material of the SKT is almost culture-free, enabling the assessment of older persons who are not native speakers in their country of residence, (3) the whole testing situation is designed more as a game than as a cognitive examination, leading to a positive affect for the subjects in the testing situation, and (4) the SKT manual is available in several languages (e.g., English, French, Italian and Spanish) [11]; the English regression-based norming will be published in 2019 .

A sample of older adults without dementia at baseline was followed for 3 years and the cognitive ability was tested with the SKT. The present study investigated the risk of developing a dementia within the next 3 years based on a person's cognitive status at baseline as rated by the SKT ("healthy," "MCI" or "suspected pathological decline").

\section{Materials and Methods}

\section{Participants}

A sample of older adults aged between 65 and 85 were recruited from the internal wards of 3 general hospitals in Munich, Germany (T0). Persons with dementia in the hospital were excluded from further examinations. Dementia was diagnosed according to the DSM-IV. Further exclusion criteria were severe hearing or visual impairment, speech impairment, or need of institutional care. The baseline assessment (T1) was conducted 3 months after discharge from the hospital; 3 follow-up assessments took place with intervals of 1 year (T2, $\mathrm{T} 3$, and T4). A detailed description of the sample and the screening procedure in the hospital is published elsewhere $[12,13]$. 


\section{Study Protocol and Measures}

The study protocol was approved by the institutional review board of the Faculty of Medicine at the Technical University of Munich (TUM; file number: 116/98). The participants were examined by trained psychiatrists and psychologists. An initial screening was conducted in the hospitals at T0. During the hospital stay, the essential instrument was the Structured Interview for the Diagnosis of Dementia of the Alzheimer Type, Multi-Infarct Dementia, and Dementias of other Etiology according to DSM-III-R, DSM-IV and ICD-10 (SIDAM) [14]. The SIDAM is a brief procedure not only for the diagnosis of dementias but also for the diagnosis of MCI. Approximately 3 months after all participants had been discharged from the general hospital and their acute illness had subsided, they were visited at their homes for a first baseline assessment (T1). At baseline the SKT and Clinical Dementia Rating [15] were applied.

The SKT, which is available in 5 parallel testing forms (form A through E), takes about 10-15 min for its administration. The test consists of 9 subtests assessing memory (3 subtests) and attention or speed of information processing (6 subtests). A detailed description of the new norming procedure is published [16]. The total summary scores range from 0 to 18. A traffic light system was developed based on optimal cutoffs for the discrimination between 3 diagnostic groups (cognitively healthy, MCI or dementia). Summary scores between 0 and 4 suggest normal cognitive aging or cognitive health (green), scores between 5 and 10 suggest MCI (yellow), and scores above 11 represent pathological cognitive decline (red).

Each participant's cognitive status was rated on the Clinical Dementia Rating Scale (CDR). The CDR discriminates between 5 stages of cognitive impairment (with corresponding numerical indices): none (0), very mild (0.5), mild (1), moderate (2), and severe (3). While a score of 0 corresponds to cognitive health and scores between 1 and 3 represent mild, moderate, and severe dementia. A score of 0.5 was conceptualized as MCI [17]. Only persons with a CDR-rating of 0 or 0.5 at baseline were included in the study. However, the participants' SKT red status (suspected pathological decline) was not an exclusion criterion. At each measurement point, each person was tested with the SKT and assessed with the CDR.

\section{Statistical Analysis}

SKT raw scores were converted into normed scores for all examinations (T1-T4). Kaplan-Meier-estimators were used to compare the cumulated risk for developing dementia during the study period between the SKT's traffic light classifications. Separate Cox-regressions were calculated to predict the onset of MCI and dementia at the 3 followup examinations. Cross-tables were built in order to determine the relationship between the SKT traffic light labels at baseline and the cognitive status according to the CDR at the 3 follow-up examinations. The SKT labels at baseline yellow or red versus green were cross-tabulated with 3 different cognitive endpoints (cognitive deterioration, MCI and dementia) at the 3 follow-up examinations. (1) "cognitive deterioration" means that the participants had after 1,2 or 3 years a CDR rating equal or greater than 0.5 . This included a change in CDR status as well from 0 to 0.5 as from 0.5 to $>0.5$. (2) "MCI" represented the incidence of MCI ( $C D R=0.5$ ) for subjects who were cognitively unimpaired at baseline. (3) "Dementia" was defined as the onset of dementia according to DSM-IV and CDR $\geq 1$. Further, cross-tables were built to map the association between SKT labels and the endpoints "healthy," "MCI" and "dementia" at the 3 follow-up examinations. Data analysis was performed with SPSS 25. 


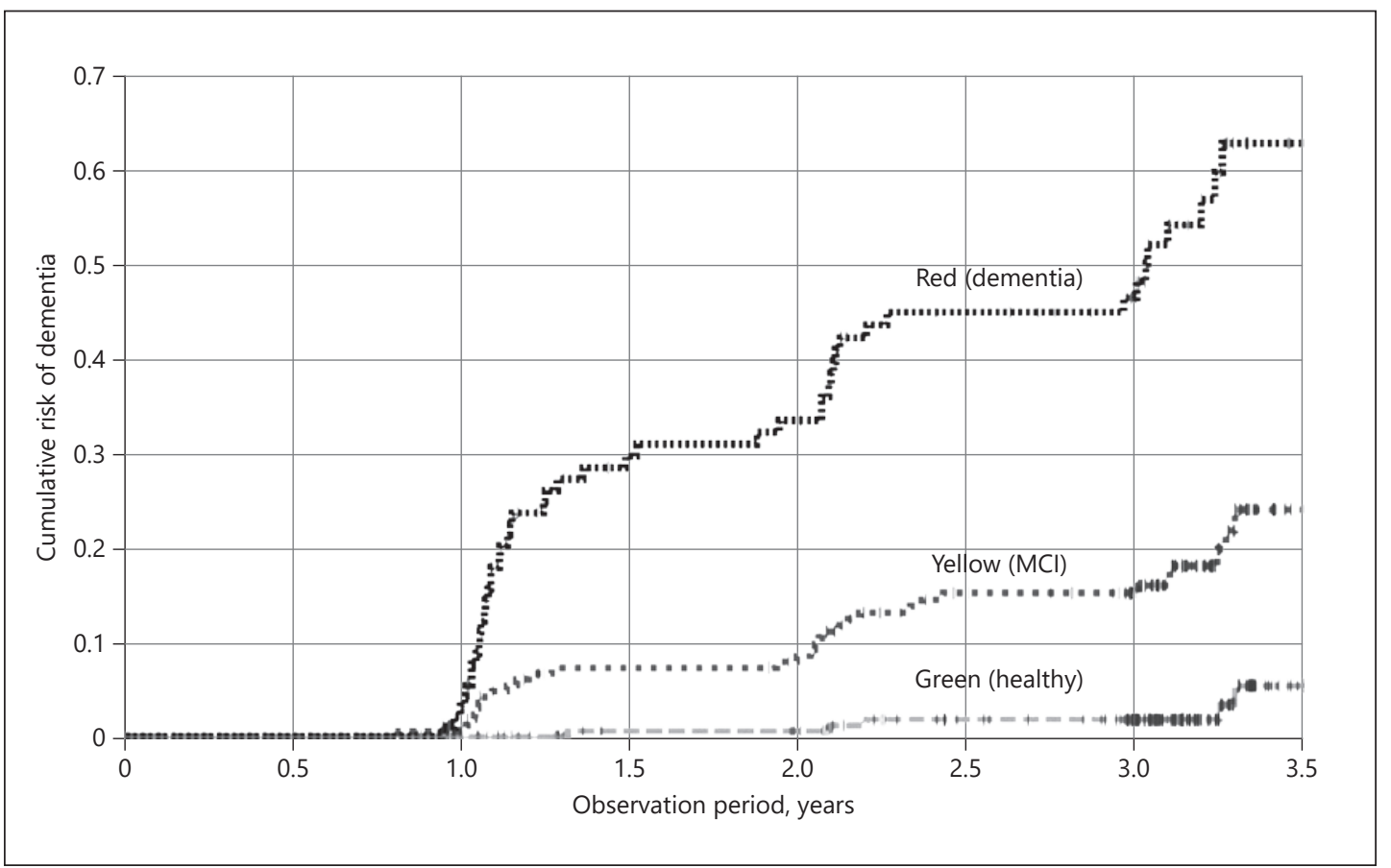

Fig. 1. Cumulative risk of dementia according to the SKT traffic light labels at baseline (Kaplan-Meier estimation). SKT, Syndrom-Kurztest; MCI, mild cognitive impairment.

\section{Results}

The sample consisted of $n=546$ persons ( $59.7 \%$ female) and the mean age was 75.7 years ( $\mathrm{SD}=5.5)$. The majority of the sample $(62.3 \%)$ had completed lower secondary education and $37.7 \%$ had higher degrees. The mean MMSE score in this sample was 26.6 (SD = 2.4). At baseline, $368(67.4 \%)$ were rated as cognitively unimpaired $(\mathrm{CDR}=0)$ according to the CDR criteria and $178(32.6 \%)$ were rated as having MCI (CDR $=0.5)$. The distribution of SKT traffic light labels at baseline was as follows: $217(40.2 \%)$ were rated as green, $205(38.0 \%)$ as yellow and $118(21.8 \%)$ as red (6 subjects had no SKT data). During follow-up 84 of the initially non-demented participants were diagnosed with a dementia, whereas 68 of the cognitively unimpaired participants developed MCI.

With regard to the total observation period (4 measurement points), $n=343$ ( $62.8 \%$ of the total sample) had data for all data assessments, 404 subjects (74.0\%) took part in at least 2 of the 3 follow-up assessments, and 458 subjects attended at least one follow-up (83.9\%). With regard to all measurement points there was an attrition of 203 participants. The attrition was due to mortality $(n=105 ; 51.7 \%)$ or due to refusal to participate $(n=85 ; 41.9 \%)$ or due to moving to another city and not being accessible anymore $(n=13 ; 6.4 \%)$. For those subjects who took part in the follow-ups, the average observation period was 2.7 years (the min-max range was between 0.8 and 4.2 years; for more details see also $[12,13])$.

Kaplan-Meier-Estimators were calculated for the risk of developing a dementia based on the SKT traffic light labels at baseline (Fig. 1). The X-axis displays the number of years under observation and the Y-axis shows the cumulated risk for dementia. The steps in each trajectory represent the onset of dementia, the short rectangular strokes represent time points at which participants dropped out of the study for any reason (e.g., refusal to participate further, death, or termination of the study). The log-rank (Mantel-Cox) test was statistically significant, $\chi^{2}=$ 
Stemmler et al.: SKT Predicting Cognitive Decline

Table 1. Risk of mild cognitive impairment, dementia, and moderate to severe dementia during follow-up in relation to the SKT traffic light labels at baseline (Cox regression)

\begin{tabular}{|c|c|c|c|}
\hline SKT traffic lights labels & $\begin{array}{l}\text { HRs for MCI } \\
(\mathrm{CDR}=0.5 ; 95 \% \mathrm{CI})\end{array}$ & $\begin{array}{l}\text { HRs for dementia } \\
(\mathrm{CDR} \geq 1 ; 95 \% \mathrm{CI})\end{array}$ & $\begin{array}{l}\text { HRs for moderate and severe } \\
\text { dementia (CDR } \geq 2 ; 95 \% \mathrm{CI} \text { ) }\end{array}$ \\
\hline Healthy (green) & Reference group & Reference group & Reference group \\
\hline MCI (yellow) & $2.34(1.37-3.99)$ & $6.63(2.75-15.96)$ & $17.23(2.26-131.19)$ \\
\hline Pathological cognitive decline (red) & $3.83(1.86-7.86)$ & $25.40(10.73-60.14)$ & $73.16(9.96-537.55)$ \\
\hline
\end{tabular}

CDR, 0.5 represents $\mathrm{MCI}$; CDR $\geq 1$ represents any dementia; CDR $\geq 2$ represents moderate and severe dementia. For MCI: $-2 \mathrm{LL}=$ 682.43 and the $\chi^{2}=17.82, \mathrm{df}=2$; for any dementia: $-2 \mathrm{LL}=848.71$ and the $\chi^{2}=117.38, \mathrm{df}=2, p<0.001$; for moderate and severe dementia: $-2 \mathrm{LL}=406.16$ and the $\chi^{2}=73.90, \mathrm{df}=2, p<0.001$. All CIs are based on 95\%. CDR, Clinical dementia rating; SKT, SyndromKurztest; HRs, hazard ratios.

117.47, $\mathrm{df}=2, p<0.001$, suggesting that the 3 trajectories are different. The time until the first diagnosis of dementia depended on the respective cognitive level at baseline. The total study period covered a total range of 3.5 years. The estimation of the cumulative risk can be reported for 3 different time spans: 1.5, 2.5, and 3.5 years. The cumulative risk of developing a dementia for the green group was 1 (1.5), 1.8 (2.5), and 5.4\% (3.5 years); for the yellow group the following cumulated risk emerged respectively: $7.3,15.2$, and $24.1 \%$. In the red group, $29.7 \%$ developed a dementia after 1.5 and $44.9 \%$ after 2.5 years, and after the last follow-up $62.8 \%$ had a diagnosis of dementia.

Cox-regressions were applied to calculate the respective hazard ratios (HRs) for the onset of dementia related to the SKT traffic light labels at baseline (Table 1). Three different clinical endpoints were used. (1) In the subsample of the cognitively unimpaired participants at baseline (CDR $=0)$, the association between the SKT traffic light labels and the risk for developing a MCI were tested, whereas in the total sample (2) the association with the onset of mild to severe dementia and (3) the association with the onset of moderate or severe dementia with regard to the SKT traffic light labels were analysed.

(1) For the prediction of MCI (CDR $=0.5$ ) based on the SKT labels the -2 log Likelihood statistic was $-2 \mathrm{LL}=682.43, \chi^{2}=17.82, \mathrm{df}=2, p<0.001$. The yellow group had a more than 2 times and the red group an almost 4 times increased risk of developing MCI during the observation period. (2) For the prediction of a dementia, the -2 log Likelihood statistic was $-2 \mathrm{LL}=848.71$, $\chi^{2}=117.38, \mathrm{df}=2, p<0.001$. The HRs for yellow was HR 6.63 and for red HR 25.40, meaning that the likelihood of developing a dementia was 6.6 times higher for the yellow group in comparison to the green group and 25.4 time higher in the red group. (3) Considering the HRs for moderate and severe dementia, the values amount to 17.23 for the yellow and 73.16 for the red group.

The relationship between the SKT traffic light labels at baseline and the cognitive status at follow-up (1,2, and 3 years later) was evaluated (Table 2). At baseline, the SKT labels yellow or red (indicating cognitive impairment) were contrasted with the label green (indicating a cognitively healthy aging) in order to look for different endpoints after different follow-up periods. Three endpoints were chosen: (1) "cognitive deterioration," (2) "MCI" and (3) "Dementia." The sensitivity, specificity, positive predictive value (PPV), and negative predictive values (NPV) were calculated.

The following analyses focused on the label green, representing healthy aging, in contrast to yellow and red which represent any cognitive impairment. For "cognitive deterioration," the sensitivity for the SKT label yellow or red was 0.93 , suggesting that of those who belonged to the participants with a cognitive deterioration after 1 year, $93 \%$ had the label yellow or red 
Table 2. Sensitivity, specificity, PPV, and NPV with regard to the SKT groups yellow/red versus green and the cognitive status at follow-up (1, 2, and 3 years later)

\begin{tabular}{|c|c|c|c|c|c|}
\hline \multirow[t]{2}{*}{ SKT traffic light labels at baseline } & \multicolumn{5}{|c|}{ CDR ratings (endpoints) } \\
\hline & $\begin{array}{l}\text { change in cognitive } \\
\text { state after }\end{array}$ & sensitivity & specificity & PPV & NPV \\
\hline \multicolumn{6}{|l|}{ Cognitive Deterioration $(C D R \geq 0.5)^{\mathrm{a}}$} \\
\hline \multirow[t]{3}{*}{ Yellow/red vs. green } & 1 year & 0.93 & 0.48 & 0.24 & 0.97 \\
\hline & 2 years & 0.82 & 0.48 & 0.28 & 0.92 \\
\hline & 3 years & 0.80 & 0.52 & 0.38 & 0.88 \\
\hline \multicolumn{6}{|l|}{$M C I(C D R=0.5)^{\mathrm{b}}$} \\
\hline \multirow{3}{*}{ Yellow/red vs. green } & 1 year & 0.92 & 0.54 & 0.29 & 0.97 \\
\hline & 2 years & 0.79 & 0.50 & 0.24 & 0.92 \\
\hline & 3 years & 0.68 & 0.55 & 0.27 & 0.88 \\
\hline \multicolumn{6}{|l|}{ Dementia $(C D R \geq 1)^{\mathrm{c}}$} \\
\hline \multirow[t]{3}{*}{ Yellow/red vs. green } & 1 year & 0.98 & 0.46 & 0.17 & 0.99 \\
\hline & 2 years & 0.95 & 0.49 & 0.23 & 0.98 \\
\hline & 3 years & 0.93 & 0.51 & 0.28 & 0.97 \\
\hline
\end{tabular}

PPV, positive predictive value; NPV, negative predictive values; SKT, Syndrom-Kurztest; CDR, clinical dementia rating scale; $\mathrm{MCI}$, mild cognitive impairment. ${ }^{\mathrm{a}}$ Cognitive Deterioration is the change in CDR status from 0 to $>0$ or from 0.5 to $>0.5$. ${ }^{\mathrm{b}} \mathrm{MCI}$ is the incidence of MCI (CDR $=0.5)$ for subjects who were cognitively unimpaired at baseline $(\mathrm{CDR}=0)$. ${ }^{\mathrm{c}}$ Dementia is the onset of a dementia according to DSM-IV and CDR $\geq 1$.

at baseline. The sensitivity declined to 0.82 after 2 years and to 0.80 after 3 years. The specificity was much lower with 0.48 , indicating that of those persons, who were not cognitively impaired after 1 year, 48\% had a green SKT label at baseline. The PPV was also low. Of those, who had an SKT label of yellow or red, 24\% belonged to the persons with a cognitive deterioration. In correspondence to the sensitivity, the NPV was high. Of those, who had a green label at baseline, $97 \%$ belonged to the group, who were not cognitively impaired (after 2 years: $92 \%$ and 3 years: $88 \%$ ).

For the endpoint MCI, the sensitivity was 0.92 (sensitivity after 2 years: 0.79 and after 3 years 0.68 ). The specificity for predicting MCI after 1 year was 0.54 . PPV was low again. Of those persons, who had a label of yellow or red at baseline, $29 \%$ belonged to the persons with MCI (PPV). Again, the NPV was high. Of those, who belonged to the green group at baseline, 97\% belonged to the group with no MCI (after 2 years: $92 \%$ and 3 years: $88 \%$ ).

For the prediction of dementia from the SKT labels at baseline, the sensitivity was 0.98 , saying that of those who belonged to the group with a dementia after 1 year, $98 \%$ had the SKT label yellow or red at baseline. Specificity was 0.46 , indicating that of those with no dementia after 1 year, $46 \%$ had the SKT label green at baseline. Again, the PPV was low and the NPV high. Of those persons, who had a label of yellow or red at baseline, $17 \%$ belonged to the persons with a dementia (PPV). Of those, who had a green label at baseline, $97 \%$ belonged to the group without dementia (after 2 years: $98 \%$ and 3 years: $97 \%$ ).

Table 3 displays the association between SKT labels at baseline and the respective cognitive status after 1,2, and 3 years. The percentages for the onset of dementia were similar. However, a significant amount of participants of the red group showed a healthy development; after 1 year, $40.4 \%$ were cognitively healthy, after 2 years, $32.1 \%$ and one-third after 3 years $(33.3 \%)$. 
Stemmler et al.: SKT Predicting Cognitive Decline

Table 3. SKT traffic light labels at baseline and cognitive status after 1,2 , and 3 years

\begin{tabular}{|c|c|c|c|c|c|c|c|c|c|c|}
\hline \multirow{2}{*}{\multicolumn{2}{|c|}{$\begin{array}{l}\text { SKT traffic light labels at } \\
\text { baseline, } \%\end{array}$}} & \multicolumn{3}{|c|}{ After 1 year, \% } & \multicolumn{3}{|c|}{ After 2 years, \% } & \multicolumn{3}{|c|}{ After 3 years, \% } \\
\hline & & healthy ${ }^{a}$ & $\mathrm{MCI}^{\mathrm{b}}$ & dementiac $^{c}$ & healthy ${ }^{\mathrm{a}}$ & $\mathrm{MCI}^{\mathrm{b}}$ & dementia $^{c}$ & healthy ${ }^{\mathrm{a}}$ & $\mathrm{MCI}^{\mathrm{b}}$ & dementia $^{c}$ \\
\hline Green & 40.2 & 96.4 & 3.1 & 0.5 & 90.6 & 7.6 & 1.8 & 85.4 & 11.8 & 2.8 \\
\hline Yellow & 38.0 & 71.4 & 21.4 & 7.1 & 66.9 & 19.5 & 13.6 & 62.9 & 21.0 & 16.1 \\
\hline Red & 21.8 & 40.4 & 30.9 & 28.7 & 32.1 & 25.6 & 42.3 & 33.3 & 15.9 & 50.8 \\
\hline
\end{tabular}

SKT, Syndrom-Kurztest; MCI, mild cognitive impairment; CDR, clinical dementia rating scale. ${ }^{a}$ Healthy participants were cognitively unimpaired $(C D R=0) .{ }^{b}$ Participants' CDR status with MCI was CDR $=0.5 .{ }^{c}$ Dementia is the onset of a dementia according to DSM-IV and CDR $\geq 1$.

\section{Discussion/Conclusion}

In the present study, the predictive validity of the newly normed SKT $[8,16]$ was investigated. The prospective study design [10] enabled the investigation of the onset of cognitive decline, including MCI and dementia (mild, moderate and severe) over a 3-year period.

For the green SKT group, it was least likely to develop a dementia within the next 3 years under investigation. With a green SKT label at baseline, the chance of developing a severe dementia was very low in the first 2 years of the study and about $5 \%$ at the end of the total study period (3.5 years).

For the yellow SKT group, the cumulative risk for developing a dementia during the study period (3.5 years) was 24\%. For the yellow group, the HR for developing an MCI was about twice as high as for the green group (i.e., the reference group) and the HR for developing a dementia was 6.6 times higher than in the green group.

For older adults with a red SKT label, the risk of developing a dementia was very high. The cumulative risk for developing a dementia within the study period was $63 \%$; compared to the green group, the risk of developing a dementia was about 25.4 times higher, and the risk of developing a moderate or severe dementia was even 73.2 times higher. In sum, the results indicated that based on the cognitive status at baseline (green, yellow, or red) different trajectories toward dementia evolved.

The newly normed SKT has proven a high predictive validity in terms of the onset of dementia. In their study in 2007, Bickel et al. [12] used the old SKT norms. Compared to the old SKT norms one can state that the new norming better predicts a dementia-free period. The old norms included many false negatives. That is, many persons who were rated as cognitively unimpaired with the old norms, showed cognitive decline.

The results have implications for a potential use of the SKT in primary care settings. Once tested with a green status, it seems that the next cognitive testing is not necessary within the next 2 and half years because the risk of developing a dementia in this period would be expected to be very low. Also, the SKT seems feasible to detect persons at high-risk for the development of dementia. In addition, the SKT may also be used to validate the status of MCI.

The sensitivity of the SKT for predicting different cognitive endpoints such as cognitive deterioration, $\mathrm{MCI}$, or dementia can be seen as very high. The sensitivity was the highest for predicting dementia. Here, even after 3 years, the sensitivity was 0.93 . In addition, belonging to the green group at baseline meant an approximately $90 \%$ chance of being cognitively unimpaired even after 3 years, no matter what the cognitive endpoints were (i.e., NPV). 
Of course, the validity statistics always depend on the sample they were calculated from and sensitivity and specificity are interdependent on each other. Therefore, high sensitivity usually corresponds to low specificity. Low specificity and low PPV are always a result of the relatively high number of false positives. Having a green label at baseline is predictive of the cognitive development within the next 3 years. Of those who belonged to the green group at baseline, $0.5 \%$ had a dementia after 1 year, $1.8 \%$ after 2 years, and $2.8 \%$ after 3 years. The new SKT norms result only in a few false negatives. In contrast, of those who belonged to the red group at baseline, $40 \%$ were cognitively unimpaired after 1 year and about one third were cognitively unimpaired after 2 and 3 years, indicating a relatively high proportion of false positives.

In a previous study on the psychometric properties of the newly normed SKT [10], we conducted ROC curves in order to examine the validity of the SKT in comparison to the MMSE to detect MCI and dementia. The AUCs for the detection of MCI ranged between 0.79 and 0.88 for the SKT and between 0.79 and 0.91 for the MMSE. For dementia, the AUCs were high and ranged between 0.91 and 0.96 for the SKT and between 0.89 and 0.95 for the MMSE. For the discrimination between $\mathrm{MCI}$ and dementia, the values ranged between 0.75 and 0.87 for the SKT and between 0.78 and 0.85 for the MMSE.

The sensitivity of the SKT for MCI was 0.92 (1 year prediction), but how do other cognitive tests predict MCI? Breton et al. [18] conducted a meta-analysis on the diagnostic accuracy in detecting MCI (a prerequisite for being selected in their study were at least 5 published studies on the diagnostic instrument). Here, the Memory Alteration Test (M@T [19]) had the highest sensitivity with 0.95 , followed by the CERAD [20] and the ACE-R [21] with 0.82. In this meta-analysis, the MMSE revealed the lowest sensitivity for MCI with 0.66.

The sample in this study was recruited from general hospitals. This may have resulted in a higher incidence of dementia than usual, which could have influenced the predictive validity of the SKT in a somewhat positive way. However, one can preclude that the overall positive predictive validity was based on any systematic measurement error because the same assessment instruments and the same diagnostic criteria were used at each time point. In sum, the psychometric properties of the SKT have proven a high predictive validity, indicating that good cognitive performance in the SKT precludes the development of dementia for some time. In addition, the SKT may be useful in prospective clinical studies, which are attempting to enroll a sample of older adults at preclinical or MCI stages who are at high risk of developing dementia within the near future. Those studies could also be used to validate biomarkers, other assessment tools, or algorithms for diagnostic purposes [22, 23].

\section{Acknowledgement}

We thank Rainer Diebow for transforming the SKT raw data into the new scale scores.

\section{Statement of Ethics}

The authors state that subjects have given their informed consent and the study protocol has been approved by the institute's committee on human research.

\section{Disclosure Statement}

M.S.: received fees for scientific advice from Geromed GmbH, Erlangen. J.B.H. and H.B.: have no conflicts of interest to declare. 


\section{Funding Source}

This study was supported by Dr. Willmar Schwabe Arzneimittel, Karlsruhe, Germany.

\section{Author Contributions}

M.S. and J.B.H.: wrote the manuscript; H.B.: did all the statistical analyses.

\section{References}

1 McKhann GM, Knopman DS, Chertkow H, Hyman BT, Jack CR Jr, Kawas CH, et al. The diagnosis of dementia due to Alzheimer's disease: recommendations from the National Institute on Aging-Alzheimer's Association workgroups on diagnostic guidelines for Alzheimer's disease. Alzheimers Dement. 2011 May; 7(3): 263-9.

2 Stemmler M, Kornhuber J. Demenzdiagnostik [Assessment of Dementia]. Göttingen: Hogrefe; 2018.

3 Anastasi A. Evolving concepts of test validation. Annu Rev Psychol. 1986;37(1):1-16.

4 Albert MS, DeKosky ST, Dickson D, Dubois B, Feldman HH, Fox NC, et al. The diagnosis of mild cognitive impairment due to Alzheimer's disease: recommendations from the National Institute on Aging-Alzheimer's Association workgroups on diagnostic guidelines for Alzheimer's disease. Alzheimers Dement. 2011;7:270-9.

5 Dubois B, Feldman HH, Jacova C, Cummings JL, Dekosky ST, Barberger-Gateau P, et al. Revising the definition of Alzheimer's disease: a new lexicon. Lancet Neurol. 2010 Nov;9(11):1118-27.

6 Morris JC, Roe CM, Grant EA, Head D, Storandt M, Goate AM, et al. Pittsburgh compound B imaging and prediction of progression from cognitive normality to symptomatic Alzheimer disease. Arch Neurol. 2009 Dec; 66(12):1469-75

7 Erzigkeit H. SKT: Kurztest zur Erfassung von Gedächtnis- und Aufmerksamkeits-störungen. Manual. Erlangen: Geromed; 2001.

8 Stemmler M, Lehfeld H, Horn R. SKT Manual. 4th extended and revised edition. Spardorf: Geromed; 2015.

9 Merten T. Über den Sinn und Unsinn der Verwendung von Screening-Instrumenten in der neuropsychologischen Diagnostik. Diagnostica. 1999;45(3):154-62.

10 Hessler JB, Stemmler M, Bickel H. Cross-Validation of the Newly-Normed SKT for the Detection of MCI and Dementia. GeroPsych (Bern). 2016 Aug;30(1):1-7.

11 Lehfeld H, Rudinger G, Rietz C, Heinrich C, Wied V, Fornazzari L, et al. Evidence of the cross-cultural stability of the factor structure of the SKT short test for assessing deficits of memory and attention. Int Psychogeriatr. 1997 Jun;9(2):139-53.

12 Bickel H, Mösch E, Förstl H. Vorhersage von Demenzerkrankungen mit dem Syndrom-Kurztest (SKT): eine Prospektivstudie. Z Gerontopsychol Psychiatr. 2007;20(1):7-16.

13 Bickel H, Mösch E, Seigerschmidt E, Siemen M, Förstl H. Prevalence and persistence of mild cognitive impairment among elderly patients in general hospitals. Dement Geriatr Cogn Disord. 2006;21(4):242-50.

14 Zaudig M, Hiller W. SIDAM-Handbuch. Strukturiertes Interview für die Diagnose einer Demenz vom Alzheimer Typ, der Multi-Infarkt-(oder vaskulären) Demenz und Demenzen anderer Ätiologien nach DSM-III-R, DSM-IV und ICD-10. Bern: Huber; 1996.

15 Hughes CP, Berg L, Danziger WL, Coben LA, Martin RL. A new clinical scale for the staging of dementia. Br J Psychiatry. 1982 Jun;140(6):566-72.

16 Stemmler M, Lehfeld H, Siebert J, Horn R. Ein kurzer Leistungstest zur Erfassung von Störungen des Gedächtnisses und der Aufmerksamkeit. Diagnostica. 2017;63(4):243-55.

17 Winblad B, Palmer K, Kivipelto M, Jelic V, Fratiglioni L, Wahlund LO, et al. Mild cognitive impairment-beyond controversies, towards a consensus: report of the International Working Group on Mild Cognitive Impairment. J Intern Med. 2004 Sep;256(3):240-6.

18 Breton A, Casey D, Arnaoutoglou NA. Cognitive tests for the detection of mild cognitive impairment (MCI), the prodromal stage of dementia: meta-analysis of diagnostic accuracy studies. Int J Geriatr Psychiatry. 2019 Feb; 34(2):233-42.

19 Rami L, Molinuevo JL, Sanchez-Valle R, Bosch B, Villar A. Screening for amnestic mild cognitive impairment and early Alzheimer's disease with M@T (Memory Alteration Test) in the primary care population. Int J Geriatr Psychiatry. 2007 Apr;22(4):294-304.

20 Chandler MJ, Lacritz LH, Hynan LS, Barnard HD, Allen G, Deschner M, et al. A total score for the CERAD neuropsychological battery. Neurology. 2005 Jul;65(1):102-6. 
21 Davies RR, Larner AJ. Addenbrookes cognitive examination (ACE) and its revision (ACE-R). In: Larner AJ, editor. Cognitive Screening Disorders. A Practical Approach. London: Springer; 2013. pp. 61-77.

22 Khachaturian ZS, Barnes D, Einstein R, Johnson S, Lee V, Roses A, et al. Developing a national strategy to prevent dementia: Leon Thal Symposium 2009. Alzheimers Dement. 2010 Mar;6(2):89-97.

23 O'Bryant SE, Lacritz LH, Hall J, Waring SC, Chan W, Khodr ZG, et al. Validation of the new interpretive guidelines for the clinical dementia rating scale sum of boxes score in the national Alzheimer's coordinating center database. Arch Neurol. 2010 Jun;67(6):746-9. 